\title{
Research on Transmission Selection Optimized Evaluation Algorithm of Multi-frequency Transmitter
}

\author{
Zhou Dandan $^{1}$, Song Jian ${ }^{1}$, Lin Changhai ${ }^{2}$, Wang Xinzhe ${ }^{2}$ \\ ${ }^{1}$ Information Science Technology College of Tsinghua University, China \\ ${ }^{2}$ Radio Film and Television Design and Research Institute, China \\ huner2011@foxmail.com
}

Keywords: multi-frequency, transmission selecting the optimized, algorithm research

\begin{abstract}
The paper starts from the research objective of multi-frequency intelligent scheduling software, and analyzes the necessity of researching transmission of multi-frequency transmitter selecting the optimized evaluation algorithm. And the appropriate evaluation algorithm is selected by algorithm comparison and data example, and the working steps and function flows are described. And the paper finally achieves the computational formula of comprehensive estimation value under the presetting conditions.
\end{abstract}

\section{Introduction}

Multi-frequency transmitter intelligent scheduling software is the architecture which not only can manage multi-frequency transmitting stations and control multi-frequency equipment, but also can rapidly change working condition of multiple devices based on considering the transmission effect of transmitters executing different tasks. The aim is to use computers to replace director of machine rooms and the person on duty to complete the above job, which not only improves the work efficiency and accuracy, but also avoids the delay and fault made by man-made operation. Transmission of multi-frequency transmitter selecting the optimized evaluation algorithm is an important part of multi-frequency transmitter intelligent scheduling software. It executes behind the scenes, and considers the relationship between multi-frequency equipment and transmitting frequency. According to the the transmission evaluation data record formed by each frequency of each transmitter selecting the optimized assessment value under different conditions, the standing-wave ratio is computed and ordered. The transmitter collects data in real time in operation, and updates continuously to ensure that the optimized selection can make the equipment with the best transmission effect complete the transmission task.

\section{Requirement Analysis}

When the running chart automatically generates and insertions the tasks, each task can select many resources. How to achieve the optimal transmission effect in the optional resources needs to judge which of the optional resources is appropriate to complete the present transmission task. The data description of the task needs to be considered, transmission frequency, transmission mode (frequency modulation, amplitude modulation and CW), power level (full, half and 1/4), start time( the breaking start time is the present time), and the end time. According to the above description, the dimension for each task selecting resources is four dimensions, frequency, mode, level and time. Combined with the requirement analysis of the transmission tasks, the transmission only uses frequency modulation and half power, so transmission mode and power level is not considered. But time has no great influence on transmission effect and resources. Therefore, the present algorithm only researches frequency as dimension parameter.

(1) Input

1)Transmit frequency band division table. It comprehensively considers the setting of transmitter, and rationally distributes transmit frequency band.

2) Equipment adjacency matrix. It expresses the adjacent relation of equipment. 
3) Forward power (P). It means the achieved forward power value by the data after conversion.

4) Backward power (r). It means the achieved backward power value by the data after conversion.

5)Threshold value. It refers to the threshold of assessment value to subset the optimized sequence of evaluation. According to the subsection, the equipment sequences are set as different levels, appropriate, pass muster and inappropriate.

(2) Output

1)Comprehensive assessment value of equipment combination (transmitter and antenna) based on transmission frequency band.

2)Comprehensive assessment value list based on transmission frequency band and equipment number.

3)Evaluation ranking list based on transmission frequency band and transmission effect of equipment.

(3) Stimulation/response sequence

Initialization is triggered by interface buttons for system managers or development dispatchers. Data maintenance and generation is triggered by communication mechanism, which means that when transmitter executes the task after collecting data.

(4)Input/output data

1)Input data. Transmitter operates and collects data (transmission frequency, forward frequency, backward frequency and transmitter number). The above data is ranked according to transmitter number and frequency band.

2) Output data. Database-transmission data record table (core data is the weighted calculation evaluation value based on forward power and standing-wave ratio), transmission selecting the optimized evaluation table (core data is rank and effect attribute).

\section{Algorithm Research}

(1) Sort algorithm and algorithm selection

Sort is the operation of ranking a series of records increasingly or decreasingly according to the size of keywords. In many ranking algorithms,insertion sort and bubble sort is called simple sort. It has no requirement on space, but the time efficiency is not stable. Compared with simple sort, rapid sort has higher requirement on space, but the time efficiency is more stable.

Inter-cut sort means to insertion a number in the ranked data sequence under the premise of ensuring the data sequence is still ordered. Bubble rank repeatedly interviews the sorted series, and it compares two elements once. Rapid sort segments the data to be ordered into two independent parts. Then, the method is used to rapidly sort the data. The sort process is recursive until the series is ordered.

The example has no high requirement on time, so simple sort can be used. And initialization function uses bubble sort, and self-learning function uses insertion sort.

(2) Algorithm process

Step 1 Initialization

1) Each equipment combination is initialized after installation and debugging, which means to select the representation frequency of each frequency band (middle frequency) to make the equipment and adjacent equipment complete the actual transmission and get the sample data. The transmitted power data of transmitters under different frequency bands is collected and converted into forward and backward power value.

2) Computing standing-wave ratio and comprehensive evaluation value. Based on the conditions with interference and without interference of adjacent equipment, the comprehensive evaluation value is used as sort basis to formulate evaluation standard.

3) When all equipment complete initialization test, all comprehensive evaluation values are recorded. The parameters of two-dimension table are frequency band and equipment number.

4) Initializing evaluation sort table. The frequency band is the dimension to establish 
one-dimension table, and the data is equipment number which is sorted according to comprehensive evaluation value.

(5) Determining calling conditions. After debugging all equipment, the initialized program is started.

Step 2 Self-learning

1) According to each transmission of equipment, the forward power and backward power value after conversion of sample data is achieved. Combined with the working condition of adjacent equipment, the comprehensive evaluation value is computed.

2) According to evaluation rules, the optimized evaluation data table of transmitter under different frequency bands is computed and saved, which generates the optimized sort table of transmitter based on different frequency bands.

3) According to comprehensive evaluation values, the comprehensive evaluation value list and evaluation sort table is verified and is used for the following computation.

4) Call condition is determined. The $\mathrm{N}$ sample after each transmission is used as the basis, and the transmission of adjacent equipment is referred to call self-learning function.

Step 3 Computing sort sequence

1) According to the parameters, the optimized equipment selection sequence of the present task is computed, and the input parameters include frequency, and the output result is equipment number sequence.

2) Determining call conditions. The running chart is used to automatically generate algorithm. The parameters are used to provide equipment sort sequence for the above algorithm.

\section{Examples of Basic Data}

(1) Transmission frequency band division table. According to the elements of a transmitter, the frequency band division is as follows.

Table 1 Transmission frequency band division table

\begin{tabular}{|l|l|l|l|l|l|l|l|l|}
\hline $\begin{array}{l}\text { Frequeny } \\
\text { band }\end{array}$ & 1 & 2 & 3 & 4 & 5 & 6 & 7 & 8 \\
\hline $\begin{array}{l}\text { Starting } \\
\text { frequency }\end{array}$ & 3.2 & 4.2 & 5.5 & 7.2 & 9.5 & 12.5 & 16.5 & 21.8 \\
\hline $\begin{array}{l}\text { End } \\
\text { frequency }\end{array}$ & 4.2 & 5.5 & 7.2 & 9.5 & 12.5 & 16.5 & 21.8 & 26.1 \\
\hline
\end{tabular}

(2) Equipment adjacency matrix.

we can get the equipment adjacency matrix of the other expression (transmitter number is the index).When the program is implemented, the distance is generally used for expression. For example, meter is the unit, which can flexibly set the adjacent threshold values, as follows.

Table 2 Transmission frequency band division table

\begin{tabular}{|l|l|l|l|l|l|l|l|l|l|l|l|}
\hline & $1 \#$ & $2 \#$ & $3 \#$ & $4 \#$ & $5 \#$ & $6 \#$ & $8 \#$ & $9 \#$ & $10 \#$ & $11 \#$ & \\
\hline $1 \#$ & & & & 21 & 21 & & & & & & \\
\hline $2 \#$ & & & 17 & & 21 & & & & 20 & & \\
\hline $3 \#$ & & 17 & & & 19 & & 22 & & & & \\
\hline $4 \#$ & 21 & & & & & & & 21 & & & \\
\hline $5 \#$ & 21 & 21 & 19 & & & & & & & & \\
\hline $6 \#$ & & & & & & & 30 & 29 & & & \\
\hline $8 \#$ & & & 22 & & & 30 & & & & 26 & \\
\hline $9 \#$ & & & & 21 & & 29 & & & & & \\
\hline $10 \#$ & & 20 & & & & & & & & 23 & \\
\hline $11 \#$ & & & & & & & 26 & & 23 & & \\
\hline
\end{tabular}

(3) Computational Formula

Computing standing-wave ratio needs to fully consider the conditions of transmitters of 
adjacent antenna. For example, the standing-wave ratio is different when the transmitter of adjacent antenna works in adjacent frequency bands or far frequency bands, different weighted parameters should be used for computation. According to the data in transmission evaluation data record table and the evaluation value of the same frequency band, the transmitters are sorted to form transmission selecting optimized evaluation table. And the effect property is achieved according to evaluation value. The optimized evaluation table is completed in transmitter. In operation, the it updates automatically according to the real-time collected data by transmitters, which means to compute the collected transmission data and remedy the information in transmission data record. Combined with the information, the sort and effect attribute of transmission selecting optimized evaluation table is adjusted.

Computing comprehensive evaluation value uses weighted sum for computation, and the benchmark data is standing-wave ratio.

Computational formula of standing-wave ratio.

$$
S W R=\frac{\sqrt{R}+\sqrt{r}}{\sqrt{R}-\sqrt{r}}
$$

For the interference conditions of adjacent equipment, when the standing-wave ratio of the equipment is 1 , and the standing-wave ratio of adjacent equipment is 22 (1..n) , and that of the non-adjacent equipment is 3 (1. . n ) , the weighted proportion is $50 \%, 40 \%$ and $10 \%$.

Comprehensive evaluation value can be expressed as

Vswr $1 \times 50 \%+\frac{\sum_{1}^{n} \text { Vswr } 2}{n} \times 40 \%+\frac{\sum_{1}^{n} \text { Vswr } 3}{n} \times 10 \%$

\section{Conclusions}

The paper explores the generation, self-learning and application method of basic data of transmission optimization, which provides data support for intelligent generation, intelligent selection algorithm for running chart. In the research and system construction in the future, how to accurately optimize evaluation data and how to use more reference dimensions to make evaluation more accurate needs to be considered. Besides transmission mode and transmission power levels, the influence of weather, time and seasons on transmission optimization can be considered.

In addition, the interference between adjacent equipment needs to be researched. The problems if the present conditions (similar frequency and far frequency) can be refined into complicated scenes and if the average proportion of the present weight is reasonable need to be deeply researched.

\section{References}

[1] Low-power and short-wave experiment launcher engineering technical specification ([2009]131).

[2] Safely transmitting automatic control platform technical specification [S], National Broadcast Film TV Ratio Station Authority.

[3] Haihua Electric Enterprise (China) Limited CompanyLF2001Operation Instruction.

[4] Zhang Haipan, Software Engineering [M], Tsinghua University Press, 2010.

[5] [US], Stanley B. Lippman, Li Shixian, C++ Primer (the fourth edition), The People's Post and Telecommunication Press, 2006. 